\title{
Long-term Cognitive and Behavioral Therapies, Combined with Augmentative Communication, are Related to Uncinate Fasciculus Integrity in Autism
}

\author{
Matteo Pardini • Maurizio Elia • Francesco G. Garaci • \\ Silvia Guida $\cdot$ Filadelfo Coniglione $\cdot$ Frank Krueger • \\ Francesca Benassi · Leonardo Emberti Gialloreti
}

(C) Springer Science+Business Media, LLC 2011

\begin{abstract}
Recent evidence points to white-matter abnormalities as a key factor in autism physiopathology. Using Diffusion Tensor Imaging, we studied white-matter structural properties in a convenience sample of twenty-two subjects with low-functioning autism exposed to long-term augmentative and alternative communication, combined with sessions of cognitive and behavioral therapy. Uncinate fasciculus structural properties correlated significantly with therapy length and early onset, as well as to clinical outcome, independently from IQ, age or symptoms severity at therapy onset. Moreover, adherence to therapy was linked with better clinical outcome and uncinate fasciculus structural integrity. The results point to the capability of a long-term rehabilitation of subjects with low-functioning autism to produce white-matter structural modifications, which could thus play a role in the rehabilitative outcome.
\end{abstract}

M. Pardini - S. Guida

Department of Neurosciences, Ophthalmology and Genetics,

University of Genoa, Genoa, Italy

M. Pardini

Magnetic Resonance Research Centre on Nervous System

Diseases, University of Genoa, Genoa, Italy

M. Pardini · F. Benassi - L. Emberti Gialloreti

Centre for Communication and Neurorehabilitation

Research-CNAPP, Rome, Italy

M. Elia

Unit of Neurology and Clinical Neurophysiopathology,

Scientific Institutes for Research, Hospitalization and Health

Care (IRCCS) Oasi Maria SS, Troina, EN, Italy

F. G. Garaci

Department of Diagnostic Imaging and Interventional

Radiology, Rome Tor Vergata University, Rome, Italy
Keywords Diffusion Tensor Imaging · Autism · Uncinate fasciculus $\cdot$ Cognitive therapy $\cdot$ Long-term rehabilitation

\section{Introduction}

Autism is a neurodevelopmental disorder characterized by communication deficits, social interaction impairments, stereotyped behaviors, and narrowed interests (American Psychiatric Association 2000). Despite the current limited understanding of the neural bases of autism, different interventions have been employed to reduce autism symptoms severity, albeit with limited effectiveness (Howlin et al. 2009). Recently, the search of the neural basis of autism has been focused on the role of white matter altered maturation (Courchesne 2004) and structural and functional

\footnotetext{
F. Coniglione

Department of Anaesthesiology and Intensive Care Medicine,

Tor Vergata University, Rome, Italy

F. Krueger

Department of Molecular Neuroscience,

George Mason University, Fairfax, VA, USA

F. Krueger

Cognitive Neuroscience Section, National Institute of Neurological Disorders and Stroke-National Institutes of Health, Bethesda, MD, USA

L. Emberti Gialloreti $(\bowtie)$

Department of Public Health and Cell Biology,

Rome Tor Vergata University, Rome, Italy

e-mail: leonardo.emberti.gialloreti@uniroma2.it
} 
connectivity abnormalities (Just et al. 2004; Pardini et al. 2009) in autism physiopathology (Muller 2007).

White matter organization can be probed in vivo with Diffusion Tensor Imaging (DTI), a brain MRI technique that measures the physical restraints to the motion of water molecules (Pierpaoli and Basser 1996). DTI can be used to extract quantitative indices of white matter structural organization, such as Fractional Anisotropy (FA), a measure that is influenced by fiber density, axonal diameter, and myelination. FA yields values between 0 and 1 ; overall, higher FA values are found in more organized white matter tracts (Pierpaoli and Basser 1996). DTI techniques have successfully been used to reveal white matter abnormalities in subjects with autism in different brain structures such as frontal and temporal fiber bundles and the corpus callosum, as well as to shed light on the relationship between the severity of autistic symptomatology and the extent of white matter abnormalities (Bloemen et al. 2010; Fletcher et al. 2010; Muller 2007; Pardini et al. 2009; Pugliese et al. 2009; Shukla et al. 2011).

In this study, we employed DTI to investigate the effects on white matter architecture of prolonged exposure to a long-term intervention protocol in autistic individuals based on augmentative and alternative communication approaches (AAC) combined with sessions of behavioral and/or cognitive therapies. These approaches represent widely used techniques in the treatment of autism and they have been shown to be effective in improving autistic subjects' quality of live through enhancement of attentive, linguistic and social skills. In fact, AAC aims to help subjects with autism to reduce attentional, memory and communication problems (Lloyd et al. 1997), while the goal of modified behavioral and cognitive therapies is usually to improve social skills and to reduce anxiety and problematic behaviors (Kendall 2006; Lang et al. 2010).

As white matter maturation is known to be completed in late adolescence (Ben Bashat et al. 2005) and to be modulated by the acquisition of cognitive skills (Mabbott et al. 2009), we propose that evaluating its integrity could represent a theoretically-sound approach to verify the effect of therapeutic interventions in autistic individuals.

\section{Methods}

\section{Subjects}

In our retrospective study we included twenty-two lowfunctioning, right-handed, non-verbal autistic males, enrolled between 2002 and 2007 from those undergoing long-term rehabilitation in the Centre for Communication and Neurorehabilitation Research-CNAPP (Rome, Italy). Clinical and demographical characteristics are reported in
Table 1. Before 2005, diagnosis of autism was performed by trained child neuro-psychiatrists and psychologists through clinical assessment, standard clinical interview on developmental history, and by observing the current functioning of the person. The diagnosis was based on DSM-IV-TR criteria (American Psychiatric Association 2000) and had to be confirmed by a second clinical assessment performed by a child neuro-psychiatrist of the National Public Health System. To be eligible for the study, subjects had to be non-verbal and have a DSM-IVTR diagnosis of Autistic Disorder. Both verbal subjects (including subjects with Asperger's Disorder) and subjects with identifiable causes of autism as well as known neurological comorbidities were excluded. In 2005 the Autism Diagnostic Observation Schedule Italian version (ADOS) (Lord et al. 2000; Tancredi et al. 2005) and the Italian version of the Autism Diagnostic Interview-Revised (ADI-R) (Lord et al. 1994; Fagioli et al. 2005) have been validated and published. Therefore, after 2005 all subjects (including those previously diagnosed) underwent standardized assessment using the ADOS (Module 1, No Words) and ADI-R scales. All enrolled subjects had higher ADOS and ADI-R scores than the accepted cut-offs for the diagnosis of autism (Lord et al. 2000; Fagioli et al. 2005; Tancredi et al. 2005). ADI-R and ADOS scores, performed when these instruments became available in Italy, are reported in Table 1.

After describing the study, the general anaesthesia procedures and its risks, the subjects, parents and/or guardians gave written informed consent according to procedures approved by the "Tor Vergata" University Institutional Review Board. Treatment was administered according to the Helsinki declaration rules of good clinical practice and ethics.

\section{Rehabilitation Protocol}

The intervention was based upon a mix of behavioral, developmental, and educational approaches, with specific focus on providing communication modalities for persons who had no spoken language. The aim of these long-term interventions, which we called WOCE (Written Output Communication Enhancement), was to enhance cognitive, communication, and social skills, while reducing autistic symptoms and related problematic behaviors.

In the framework of the intervention, rehabilitation consisted of supporting strategies to develop intentional pointing, of fostering communication to acquire intentionality, of promoting communicative exchange with the world around, and of facilitating voluntary motor planning and pointing. Communication-focused interventions were based on AAC approaches to substitute or enhance speech (Lloyd et al. 1997), combined with adapted cognitive and 
Table 1 Demographic, clinical, and MRI characteristics of the enrolled subjects

\begin{tabular}{lcccc}
\hline & All subjects & $\begin{array}{l}\text { Moderate adherence } \\
(80-90 \% \text { of sessions })\end{array}$ & $\begin{array}{l}\text { High adherence } \\
(\geq 90 \% \text { of sessions) }\end{array}$ & $\begin{array}{l}P \text { scores (high vs. } \\
\text { moderate adherence) }\end{array}$ \\
\hline Age at MRI (years) & $21.9 \pm 0.5$ & $22.0 \pm 0.7$ & $21.5 \pm 0.5$ & $P=0.8$ \\
Age at therapy onset (years) & $15.5 \pm 0.8$ & $16.1 \pm 0.5$ & $15.1 \pm 0.1$ & $P=0.8$ \\
Therapy length (months) & $72.5 \pm 6.7$ & $69.8 \pm 4.5$ & $76.6 \pm 3.5$ & $P=0.09$ \\
CARS score at therapy onset & $40.5 \pm 0.8$ & $41.6 \pm 0.5$ & $38.1 \pm 1.2$ & $P=0.7$ \\
CARS score at MRI & $34.0 \pm 1.0$ & $33.0 \pm 1.4$ & $36.0 \pm 1.9$ & $P=0.7$ \\
CARS difference & $6.5 \pm 0.6$ & $4.9 \pm 0.6$ & $47.8 \pm 1.2$ & $P=0.001$ \\
Non verbal IQ at therapy onset & $48.9 \pm 1.6$ & $49.9 \pm 1.9$ & $23.2 \pm 1.9$ & $P=0.8$ \\
ADOS Module 1 & $22.6 \pm 2.1$ & $21.2 \pm 1.8$ & & $P=0.7$ \\
$\quad$ Communication + Social & & & $11.6 \pm 1.0$ & $P=0.7$ \\
Interaction) & $12.2 \pm 1.6$ & $12.8 \pm 1.0$ & $20.3 \pm 0.5$ & $P=0.8$ \\
ADI-R Communication non verbal & $19.3 \pm 1.5$ & $19.0 \pm 1.9$ & $5.8 \pm 2.3$ & $P=0.7$ \\
ADI-R social interaction & $5.5 \pm 2.5$ & $5.0 \pm 2.0$ & $0.64 \pm 0.01$ & $P=0.002$ \\
ADI-R- restricted, repetitive behaviours & $0.58 \pm 0.01$ & $0.55 \pm 0.01$ & \\
UF mean FA & & & \\
\hline
\end{tabular}

behavioral techniques (Kendall 2006). The AAC strategies used by the protocol included gestures, manual signing, finger spelling, selection-based methods, communication boards, and/or exchange based approaches. These approaches aimed at developing-whenever possible-attention, procedure memory, turn taking and reciprocal interaction, imitation, gesture and action, and a shared vocabulary through pictures and words (Lloyd et al. 1997).

For each participant, basic communication abilities were observed and natural communication was registered by two independent observers. The life context was described and the skills that the person did and did not have were determined. The overall goal was to help each person to develop communication skills that would enable him/her to be, in the long run, as independent as possible. The adapted cognitive and behavioral interventions were based on group therapy with a focus to train and practice social skills with peers (e.g., schoolmates, siblings). Communicating with peers and emotional control was exercised during the meetings, giving the possibility to also apply anxiety reducing techniques. Parents were continuously and actively involved with homework activities and by fostering environmental adjustments. Individual therapy sessions focused mainly on strategies to sustain communication, reduce anxiety and cope with rejection by others (Lang et al. 2010). There was a written set of instructions for the development of each skill. The behavior analyst in charge of the program trained everybody who worked with the person (e.g., school teacher, parent, therapist) to implement the same program. The intervention strategies were reviewed on a regular basis and redirected and adjusted by the behavior analyst when progress was not adequate. The aim of the sessions was to improve the subject's control over his symptoms and to facilitate behavioral modifications, while addressing both anxiety and social skill deficits (Kendall 2006).

In addition, each therapist kept track of the adherence (i.e., actual hours of therapy/foreseen hours of therapy) to the therapy through a weekly "journal of activities". Subjects were only included in this study for an adherence higher than $80 \%$.

On average, therapy took place for approximately $40 \mathrm{~h}$ per week, including interventions at the rehabilitation centre, school, and home. The intervention at the centre consisted of $2 \mathrm{~h}$ of treatment twice a week. The intervention at school (on average $25 \mathrm{~h}$ per week) focused on peer group activities, literacy, cognitive, emotional and social improvement, development of communication skills. Older subjects who did not attend school took part in daily educational opportunities organized in coordination with the staff of the rehabilitation centre. Work at home (1-2 $h$ daily) was mainly sustained by the parents, trained by the staff of the rehabilitation centre. In our opinion, it is important for parents to be involved and trained to implement the activities in various informal settings, such as home, playground, community, and sport facilities. The intervention at home focused mainly on homework, communication, and development of autonomy.

\section{Evaluation}

Childhood Autism Rating Scale (CARS) scores (Schopler et al. 1980), an autism severity measure widely used in Italian clinical settings before 2005, were collected before the enrollment in the rehabilitative protocol and at the time of the MRI. At the time of the enrollment the non-verbal IQ was assessed by applying the Leiter International Performance Scale-Revised (Leiter 1979). 
MRI Acquisition and Analysis Protocol

Autistic subjects underwent scanning under general anesthesia. Anesthesia, always with spontaneous breathing, was induced with intravenous propofol or inhalatory sevoforane. No complications occurred during or after MRI scanning. Scans were obtained on a 3T scanner (Intera Achieva, Philips Medical Systems, Best, the Netherlands) equipped with both $80 \mathrm{mT} / \mathrm{m} / \mathrm{ms}$ gradient coils, and an 8 -channel sensitivity encoding (SENSE) coil. DTI was performed using single-shot spin-echo echo-planar imaging (TR = $10,000 \mathrm{~ms} ; \mathrm{TE}=59 \mathrm{~ms}$; FLIP angle $90^{\circ}$; matrix size $112 \times 112$; FOV $224 \mathrm{~mm}$; slice thickness $2 \mathrm{~mm}$; gap between slices $=0$; Number of Signal Averages $=3$; SENSE factor $=2 ; \mathrm{b}=1,000 \mathrm{~s} / \mathrm{mm}^{2}$ ). Diffusion gradients were applied in 33 non-collinear directions; moreover, six $\mathrm{b}=0$ images were acquired for each subject.

DTI data were processed by using FDT software included in FSL software package (Smith et al. 2004). Pre-processing steps included correction for eddy current distortions and motion artifacts as described elsewhere (Pardini et al. 2009). FA parametric maps were obtained for all subjects and were realigned into a common space, using the nonlinear registration tool IRKT included in FSL (Smith et al. 2004) and then smoothed with a 4-mm FWHM isotropic Gaussian kernel (Pardini et al. 2009).

Normalized, smoothed FA maps were analyzed in SPM5 (http://www.fil.ion.ucl.ac.uk/spm/) using a voxel-wise approach. In SPM, a covariate-only analysis was performed to verify the relationship between local FA values and CARS scores difference between the evaluations at the beginning and at the end of planned therapy. To reach this aim, CARS score difference was entered in the model as regressor, while CARS scores at the beginning of therapy, IQ and subject's age at the time of MRI scanning were entered as covariates. The relationship between CARS scores differences and local FA values was assessed in SPM using one tailed t-tests assuming that higher FA values would be associated with bigger improvements in CARS scores. Correlational analysis, limited to white matter regions (identified using the white matter tissue mask included in FSL), was performed setting the voxellevel statistical threshold at $p<0.05$ FDR-corrected for multiple comparisons with an additional cluster extent threshold of 50 voxels. Significant clusters were then superimposed on the JHU white matter tractography atlas included in FSL (Smith et al. 2004) to identify the inclusion of those clusters in specific white matter tracts. Aim of this whole brain analysis was therefore to shed light on the location of those clusters related with CARS scores differences and thus to potentially identify those tracts related with the observed clinical improvement. Significant clusters were found to be included in the uncinate fasciculi
(UF); for each subject, mean FA values were then extracted from the uncinate fasciculi (UF) identified masks created using the JHU white matter tractography atlas included in FSL (Wakana et al. 2007).

\section{Statistical Analysis}

Associations between clinical and MRI variables were assessed using Pearson's correlations and partial correlations. Subjects were also divided into two subgroups according to adherence to therapy session at our center; subjects with an adherence to $80-90 \%$ of the session (i.e. who took part in $80-90 \%$ of the programmed sessions) were included in the moderate adherence group while subjects with an adherence $\geq$ of $90 \%$ were included in the high adherence group. All enrolled subjects presented with an adherence to therapy of at least $80 \%$ of the programmed sessions. Clinical and MRI data were then compared between the two groups using independent samples $t$ tests. Lastly, we used SPSS 16 (www.spss.com/statistics) to run a stepwise linear regression model using as target variable mean UF values and therapy length, CARS scores at enrollment and at the end of treatment, IQ at enrollment, adherence to therapy and age at MRI scanning as predictors. Aim of the linear regression analysis was to confirm the relationship between DTI metrics and therapy length taking into account possible confounding variables. Age of therapy onset was not included in the model given its observed high correlation with length of therapy (see below). All results are reported as means \pm standard errors.

\section{Results}

\section{Evaluation}

CARS scores are reported in Table 1. There were significant correlations between CARS scores difference and therapy length $(r=0.55 ; P=0.001)$ and age of therapy onset $(r=-0.71 ; P=0.001)$. There was also a significant correlation between therapy length and age of therapy onset $(r=-0.73, P=0.001$; $)$ while there were no significant correlations between therapy length or age of therapy onset and CARS scores at the beginning of the therapy. Moreover, there was no correlation between age of MRI scanning and CARS scores differences, therapy length or therapy onset. ADI-R and ADOS scores, performed when these instruments became available in Italy, therefore not at therapy onset for all subjects (see Methods section), are reported in Table 1. There was no correlation between therapy length or age of therapy onset and ADI-R and ADOS scores. 
Voxel-wise Analysis of FA Maps

Using a statistical threshold of $P<0.05$ FDR-corrected for multiple comparisons, voxel-wise analysis of FA maps revealed a significant relationship between CARS scores difference and local FA values in two separate clusters, as shown in Fig. 1 (cluster 1: local volume maxima according to Montreal Neurological Institute Coordinates: $-20,32$, -6 ; voxel $\mathrm{T}$ score: 9.2; cluster size: 310 . Cluster 2 local volume maxima according to Montreal Neurological Institute Coordinates $34-4-12$; voxel $\mathrm{T}$ score: 9.6; cluster size: 280). Both clusters were found to be included in the left and right UF, respectively, as shown in Fig. 1. Mean FA values inside the bilateral UF mask, were then calculated for each subject and used in the successive statistical analyses.

\section{DTI Metrics and Behavioral Data}

UF mean FA values are reported in Table 1. The correlation between CARS scores difference and UF mean FA values was significant (Fig. 2; $r=0.71 ; P=0.001$ ) and it remained significant after controlling for CARS scores at enrollment, age of therapy onset, age at MRI and IQ $(r=0.65 ; P=0.001)$, using a partial correlation approach.

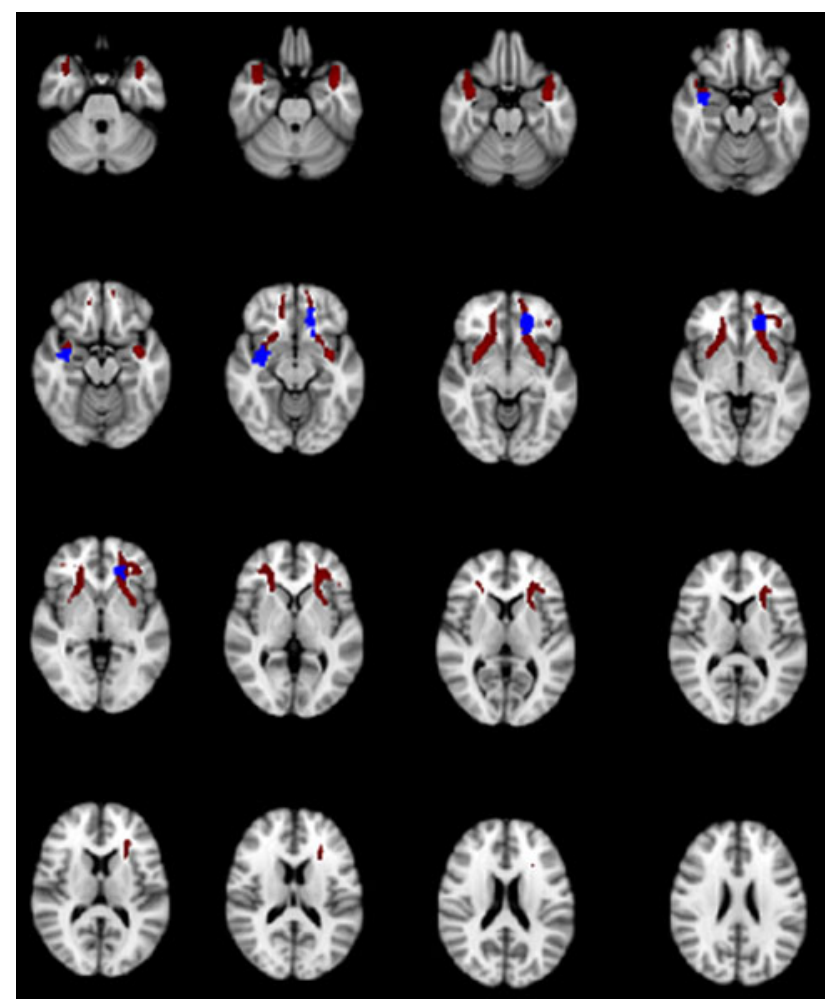

Fig. 1 Voxel-wise correlation of local FA values and CARS scores differences. Significant voxels are shown in blue, UF masks are shown in red. Images are in radiological convention
There was no significant correlation between age at the time of MRI scanning and UF mean FA values. Moreover, there was no correlation between UF mean FA values ADI-R and ADOS scores.

\section{DTI Metrics and CBT}

A significant correlation was found between UF mean FA and therapy duration (Fig. $2 ; r=0.80, P<0.001$ ) and age of therapy onset (Fig. $2 ; r=-0.77, P<0.001$ ). The correlations between UF mean FA values and therapy duration $(r=0.75 ; P=0.001)$ and age of therapy onset $(r=-0.74 ; P=0.001)$ remained significant also after controlling for CARS scores at enrollment, age at the time of MRI scanning and IQ, using a partial correlation approach.

\section{Moderate Versus High Adherence Groups}

Mean adherence to therapy was $88 \pm 4.2 \%$ of the planned in-centre sessions. Subjects in the high adherence group (12 subjects, mean adherence to therapy: $91 \pm 1.2 \%$, range 90-95\%) showed a significantly higher CARS scores difference (Fig. 3, $P=0.001$ ) and UF mean FA values (Fig. 2, $P=0.005$ ) compared to moderate adherence group (10 subjects, mean adherence to therapy: $84 \pm$ $1.4 \%$, range $80-85 \%$ ). There were no group differences in initial CARS scores, pre-treatment IQ, age of therapy onset, ADI and ADOS scores.

\section{Linear Regression of UF Mean FA Values}

The stepwise linear regression analysis of UF mean FA values using the predictor variables described in the Methods section revealed a significant model $(F=54.1$, $P<0.001$, adjusted $\mathrm{R}$ square $=0.72$ ) that explained more than $70 \%$ of the observed variance in UF mean FA values. The variables retained as significant in the model were therapy length $(P<0.001$, beta $=0.9)$ and therapy adherence $(P=0.001$, beta $=0.65)$; the other variables were not retained in the model.

\section{Discussion}

In this study we investigated the use of DTI to explore the impact of a long-term intervention protocol based on augmentative and alternative communication approaches combined with sessions of cognitive and behavioral therapy on white matter architecture in autistic individuals. Firstly, using a voxel-wise approach, we showed a significant relationship between mean FA values of two white matter clusters included in the UF and clinical improvement, 
Fig. 2 Scatterplots of autistic subjects uncinate fasciculi mean FA values and CARS scores difference (panel a), therapy length (panel b), and age of therapy onset (panel c)
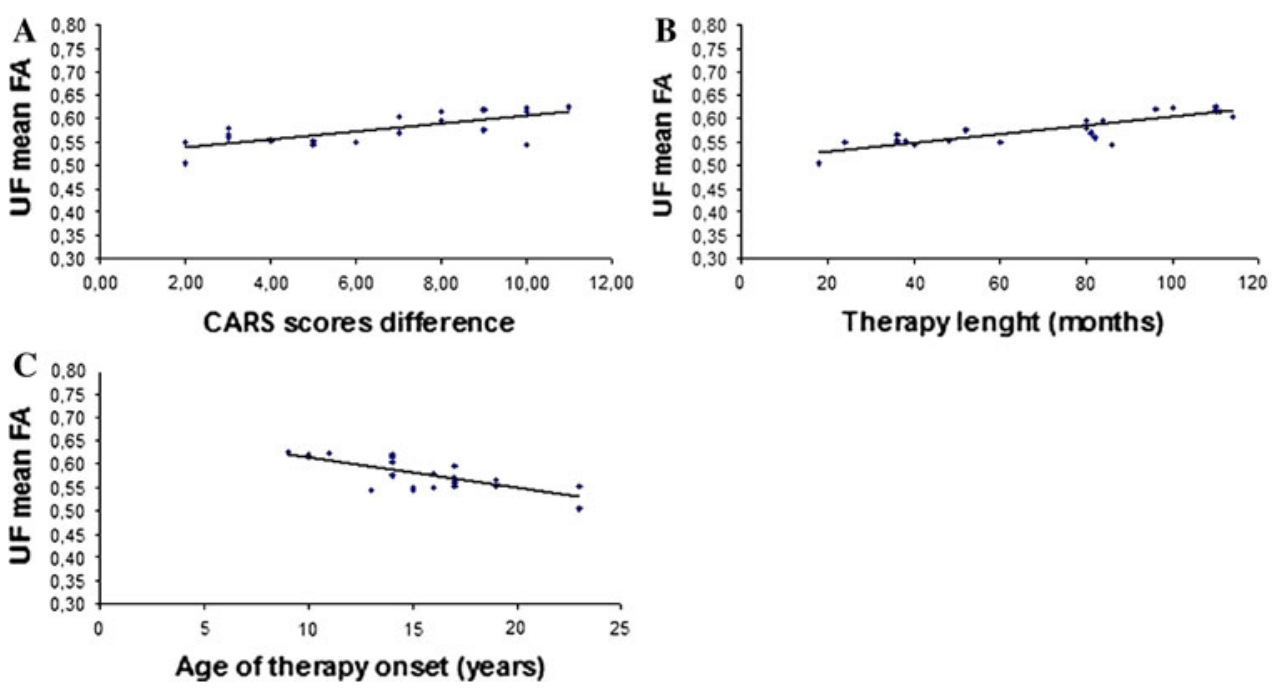
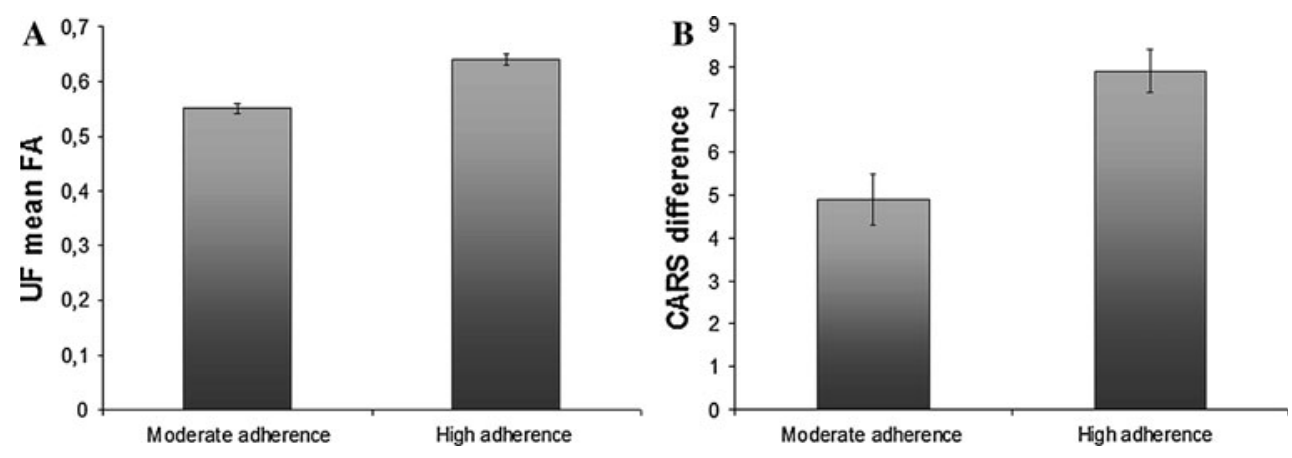

Fig. 3 Uncinate fasciculi mean FA values (panel a) and CARS scores difference (panel b), for the Moderate adherence and High adherence groups independently from symptoms severity and IQ at therapy onset and from subject's age at the time of MRI scanning. Moreover, independently of symptoms severity and IQ scores, we observed significant association between the increase of UF structural organization and the clinical improvement, precocity, and length of the intervention. Coherently with these findings we showed that subjects with a high adherence to therapy presented with a more significant clinical improvement and higher UF mean FA values, compared to the moderate adherence group.

Lastly, using a linear regression approach, we showed that length of therapy and treatment adherence explained together more than $70 \%$ of the observed UF mean FA variance, independently from subject's age at MRI scanning, IQ at therapy onset, and symptoms severity both at therapy onset, and at the end of therapy.

The UF is the main ventral limbic tract that connects frontal and temporal territories, i.e., those grey matter structures that are thought to play a key role in autism (Muller, 2007; Waiter et al. 2004). Moreover, severe socio-emotional deprivation has been shown to impact UF maturation and to be correlated with reduced uncinate fasciculus mean FA values (Eluvathingal et al. 2006). Lastly, UF maturation has been linked with the acquisition of verbal language in DTI studies (Mabbott et al. 2009) and it is thought to play a significant role in emotion expression (Schmahmann et al. 2008).

While, given the moderate size of our population, we cannot exclude that also other tracts can be modulated by AAC interventions in autism. The aforementioned observations of a specific role of the UF in those cognitive and emotional domains altered in autism, its function as main limbic connection between frontal and temporal territories, and its modulation by environmental stimuli such as social deprivation (Eluvathingal et al. 2006) seem to be consistent with our observations. UF mean FA could thus potentially represent a sensitive MRI marker of treatment-related white matter architectural modifications; future studies are needed to verify this hypothesis.

Besides structural alterations in the UF, other white matter tracts are known to be altered in autistic subjects. Indeed, different studies have shown diffuse white matter alterations in autistic subjects in structures such as the corpus callosum, the cingulum, the fornix, the arcuate fasciculus, the thalamic radiations and the superior longitudinal fasciculi (Bloemen et al. 2010; Fletcher et al. 2010; Muller 2007; Pardini et al. 2009; Pugliese et al. 2009; Shukla et al. 2011). While our whole brain voxel-wise 
correlation of local FA values with clinical improvement was only significant for the UF, studies with larger experimental populations, and, therefore, with higher statistical power, are warranted to verify the possible effect of rehabilitative interventions on other white matter structures.

Note that the aim of the study was not to show a selectivity of the correlation between rehabilitative interventions and specific structures, but instead to evaluate the use of DTI metrics as an intermediate phenotype of response to therapy in low-functioning autism. Several authors have shown the capability of behavioral interventions in altering cortical connectivity. Keller and Just observed how intensive remedial instruction resulted in increased FA of poor readers (Keller and Just 2009). More recently, Spironelli and collegues suggested that phonological training might reorganize cortical areas in dyslexic children (Spironelli et al. 2010). Furthermore, cognitive behavioral therapy (CBT) techniques have been correlated with neuroplastic changes in different pathological conditions using neuroimaging techniques (Linden 2006; de Lange et al. 2008). The potential of MRI techniques to measure CBT impact on brain architecture has been shown also in a recent study on chronic fatigue syndrome patients, in which CBT has been correlated with prefrontal cortex gray matter modulation (de Lange et al. 2008).

Our data suggest that early and prolonged treatment, as well as high adherence to the therapeutic process are instrumental to achieve a better clinical outcome. In fact, the high correlation between onset and duration of treatment makes it difficult to say whether age of onset has an effect independent of duration of treatment on the outcome.

Nevertheless, early rehabilitative interventions have been recently related to a better functional outcome in a subset of autistic subjects (Howlin et al. 2009). This observation seems to be in line with our finding of an association between age at therapy onset and UF increased structural integrity. One possible application of MRI techniques to treatments of autistic subjects could be the individuation of subsets of patients that could better respond to specific treatment approaches; future studies are warranted to explore this possibility.

Our findings need to be interpreted with caution, since our study was not a randomized clinical trial but an observational study based on a retrospective design of a small sample of low-functioning autistic subjects. Moreover, the results of our study could be strengthened by the application of DTI techniques at the beginning of the therapeutic process to better characterize the causality link between DTI metrics and therapy and, therefore, support the proposed interpretation of our data. It should also be highlighted that the presence of baseline DTI data could be used to verify if there is a link between pre-treatment mean FA values and treatment effectiveness. Such an alternative interpretation could, theoretically, lead to tailor the treatment protocol on the characteristics of each treated autistic individual.

However, when the majority of enrolled subjects started their rehabilitation, DTI acquisition and analysis were not commonly available. In addition, the need of anesthesia to perform MRI studies seemed to be an excessive risk in underage subjects in absence of preliminary data on efficacy of the proposed therapeutic intervention. Lastly, the lack of a control group of non-treated autistic subjects should lead to caution in the interpretation of these results. However, the absence of a non-treated autistic group is due to the fact that there are no young adults with autism who never underwent some sort of intervention during their childhood. We thus tried to partially compensate for the lack of a control group by comparing subjects with high and moderate adherence to the rehabilitative protocol. Nevertheless, given the positive nature of our findings more studies are now warranted.

One of the novel aspects of our study was the focus on low-functioning autistic individuals. To date, most published imaging studies are limited to high-functioning autistic subjects (Muller 2007; Pardini et al. 2009). While both the MRI data acquisition and therapeutic interventions can be challenging in this population, we feel that the relative lack of studies focused on low-functioning subjects prompts for a significant effort from the scientific community to better characterize the clinical and morphological characteristics of this difficult-to-treat population. Prospective, larger studies are needed to confirm such findings and to explore the possible clinical application of DTI in treatment efficacy studies.

Conflict of interest The authors report no conflict of interest.

\section{References}

American Psychiatric Association. (2000). Diagnostic and statistical manual of mental disorders, text revision (4th ed.). Washington, DC: American Psychiatric Association.

Ben Bashat, D., Ben Sira, L., Graif, M., Pianka, P., Hendler, T., Cohen, Y., et al. (2005). Normal white matter development from infancy to adulthood: Comparing diffusion tensor and high $b$ value diffusion weighted MR images. Journal of Magnetic Resonance Imaging JMRI, 21(5), 503-511.

Bloemen, O. J., Deeley, Q., Sundram, F., Daly, E. M., Barker, G. J., Jones, D. K., et al. (2010). White matter integrity in Asperger syndrome: A preliminary diffusion tensor magnetic resonance imaging study in adults. Autism research: Official Journal of the International Society for Autism Research, 3(5), 203-213.

Courchesne, E. (2004). Brain development in autism: Early overgrowth followed by premature arrest of growth. Mental Retardation and Developmental Disabilities Research Reviews, 10(2), 106-111.

de Lange, F. P., Koers, A., Kalkman, J. S., Bleijenberg, G., Hagoort, P., van der Meer, J. W., et al. (2008). Increase in prefrontal 
cortical volume following cognitive behavioural therapy in patients with chronic fatigue syndrome. Brain A Journal of Neurology, 131(Pt 8), 2172-2180.

Eluvathingal, T. J., Chugani, H. T., Behen, M. E., Juhasz, C., Muzik, O., Maqbool, M., et al. (2006). Abnormal brain connectivity in children after early severe socioemotional deprivation: A diffusion tensor imaging study. Pediatrics, 117(6), 2093-2100.

Fagioli, R., Saccani, M., Persico, A. M., Tancredi, R., Parrini, B., \& Igliozzi, R. (2005). Autism diagnostic interview-revised. Italian version. Firenze, Italy: Giunti O. S.

Fletcher, P. T., Whitaker, R. T., Tao, R., DuBray, M. B., Froehlich, A., Ravichandran, C., et al. (2010). Microstructural connectivity of the arcuate fasciculus in adolescents with high-functioning autism. Neuroimage, 51(3), 1117-1125.

Howlin, P., Magiati, I., \& Charman, T. (2009). Systematic review of early intensive behavioral interventions for children with autism. American Journal on Intellectual and Developmental Disabilities, 114(1), 23-41.

Just, M. A., Cherkassky, V. L., Keller, T. A., \& Minshew, N. J. (2004). Cortical activation and synchronization during sentence comprehension in high-functioning autism: Evidence of underconnectivity. Brain A Journal of Neurology, 127(Pt 8), $1811-1821$.

Keller, T. A., \& Just, M. A. (2009). Altering cortical connectivity: Remediation-induced changes in the white matter of poor readers. Neuron, 64, 624-631.

Kendall, P. C. (2006). Child and adolescent therapy: Cognitivebehavioral procedures. New York, NY: Guilford Press.

Lang, R., Regester, A., Lauderdale, S., Ashbaugh, K., \& Haring, A. (2010). Treatment of anxiety in autism spectrum disorders using cognitive behaviour therapy: A systematic review. Developmental Neurorehabilitation, 13(1), 53-63.

Leiter, R. G. (1979). Instruction manual for the leiter international performance scale. Wood Dale, Il: Stoelting Co.

Linden, D. E. (2006). How psychotherapy changes the brain-the contribution of functional neuroimaging. Molecular Psychiatry, 11(6), 528-538.

Lloyd, L. L., Fuller, D. R., \& Arvidson, H. H. (1997). Augmentative and alternative communication: A handbook of principles and practices. Needham Heights, MA: Allyn \& Bacon.

Lord, C., Rutter, M., \& Le Couteur, A. (1994). Autism diagnostic interview-revised: A revised version of a diagnostic interview for caregivers of individuals with possible pervasive developmental disorders. Journal of Autism and Developmental Disorders, 24(5), 659-685.

Lord, C., Risi, S., Lambrecht, L., Cook, E. H., Leventhal, B. L., DiLavore, P. C., et al. (2000). The autism diagnostic observation schedule-generic: A standard measure of social and communication deficits associated with the spectrum of autism. Journal of Autism and Developmental Disorders, 30(3), 205-223.

Mabbott, D. J., Rovet, J., Noseworthy, M. D., Smith, M. L., \& Rockel, C. (2009). The relations between white matter and declarative memory in older children and adolescents. Brain Research, 1294, 80-90.
Muller, R. A. (2007). The study of autism as a distributed disorder. Mental Retardation and Developmental Disabilities Research Reviews, 13(1), 85-95.

Pardini, M., Garaci, F. G., Bonzano, L., Roccatagliata, L., Palmieri, M. G., Pompili, E., et al. (2009). White matter reduced streamline coherence in young men with autism and mental retardation. European Journal of Neurology the Official Journal of the European Federation of Neurological Societies, 16(11), 1185-1190.

Pierpaoli, C., \& Basser, P. J. (1996). Toward a quantitative assessment of diffusion anisotropy. Magnetic Resonance in Medicine Official Journal of the Society of Magnetic Resonance in Medicine/Society of Magnetic Resonance in Medicine, 36(6), 893-906.

Pugliese, L., Catani, M., Ameis, S., Dell'Acqua, F., Thiebaut de Schotten, M., Murphy, C., et al. (2009). The anatomy of extended limbic pathways in Asperger syndrome: A preliminary diffusion tensor imaging tractography study. Neuroimage, 47(2), 427-434.

Schmahmann, J. D., Smith, E. E., Eichler, F. S., \& Filley, C. M. (2008). Cerebral white matter: neuroanatomy, clinical neurology, and neurobehavioral correlates. Annals of the New York Academy of Sciences, 1142, 266-309.

Schopler, E., Reichler, R. J., DeVellis, R. F., \& Daly, K. (1980). Toward objective classification of childhood autism: Childhood autism rating scale (CARS). Journal of Autism and Developmental Disorders, 10(1), 91-103.

Shukla, D. K., Keehn, B., \& Muller, R. A. (2011). Tract-specific analyses of diffusion tensor imaging show widespread white matter compromise in autism spectrum disorder. Journal of Child Psychology and Psychiatry and Allied Disciplines, 52, 286-295.

Smith, S. M., Jenkinson, M., Woolrich, M. W., Beckmann, C. F., Behrens, T. E., Johansen-Berg, H., et al. (2004). Advances in functional and structural MR image analysis and implementation as FSL. Neuroimage, 23(Suppl 1), S208-S219.

Spironelli, C., Penolazzi, B., Vio, C., \& Angrilli, A. (2010). Cortical reorganization in dyslexic children after phonological training: Evidence from early evoked potentials. Brain, Advance Access published August, 5, 2010.

Tancredi, R., Saccani, M., Persico, A. M., Parrini, B., Igliozzi, R., \& Fagioli, R. (2005). Autism diagnostic observation schedule Italian version. Firenze, Italy: Organizzazioni Speciali.

Waiter, G. D., Williams, J. H., Murray, A. D., Gilchrist, A., Perrett, D. I., \& Whiten, A. (2004). A voxel-based investigation of brain structure in male adolescents with autistic spectrum disorder. Neuroimage, 22(2), 619-625.

Wakana, S., Caprihan, A., Panzenboeck, M. M., Fallon, J. H., Perry, M., Gollub, R. L., et al. (2007). Reproducibility of quantitative tractography methods applied to cerebral white matter. Neuroimage, 36(3), 630-644. 